\title{
Quality in a psychotherapy service
}

\section{A review of audiotapes of sessions}

\author{
F. Denman
}

\begin{abstract}
General audit of psychotherapy services is stowly becoming a more regular part of practice in psychotherapy departments. Parry (1992) in her key reviow article states that "unmonitored practice is no longer defensible". Audit specifically targeted at the quality of psychotherapy performed has been recommended (Fonagy a Higgitt, 1989) but it is not often reported in psychotherapy (although a notable exception is Feldmon, 1992). The beneficial effects of an ad hoc audit of the quality of psychotherapy using tape recorded sessions are reported.
\end{abstract}

The department of psychotherapy under study offered brief focal psychotherapy conducted by trainees under supervision to a wide range of out-patients referred by psychiatrists from a general clinic without further assessment or selection. The therapeutic modality used was cognitive analytic therapy (CAT) a brief focal therapy devised by Ryle (1990) which aims to combine both cognitive and psychoanalytic elements.

Audit for outcome and process had previously been conducted within the department of psychotherapy (Watson \& Ryle, 1992) but audit for quality had not so far been attempted. At the time of the audit there were a number of concerns about the quality of the service centred on patient selection, on selection of an appropriately qualified therapist to treat the patient, on the quality of the therapy delivered and on the quality of the supervision and training offered.

\section{The study}

Eighteen randomly selected tape recordings of single sessions taken from the mid point of CAT therapies were reviewed for a number of quality indicators by two pairs of raters. Eight features were rated in a semi-quantitative way as present or absent (see Table 1).

Raters negotiated a consensus agreement about each rating and they also prepared a three line report on the quality of each tape.

\section{Findings and comment}

We looked first at the general characteristics of the sample. Most therapists were categorised as having either psychodynamic $(n=6)$ or cognitive $(n=7)$ backgrounds or pure CAT backgrounds $(n=3)$, and most were trainees $(n=13)$. Most $(n=11)$ of the patients treated fell into the standard category. We looked at the quality of the general, cognitive and psychodynamic aspects of the therapy. An important general therapeutic skill is the empathy required to pick up and respond appropriately to the predominant emotional tone of the session. Worryingly we found seven tapes where the affective tone of the patient was obviously missed.

The items which measured feedback and interpretation type used looked mainly at the cognitive aspects of CAT delivery because both are concerned with ensuring the accurate transmission of an idea. We hoped to find many definite interpretations and a high level of feedback. The majority of therapists were found to use both vague and definite interpretations $(n=10)$. Sadly, four therapists used no definite interpretations at all which means that during the session nothing was directly linked to the focus of therapy. Feedback was used only by just over half the therapists. This is a disappointing figure, particularly because the raters' impression from listening to the tapes was that there were many communicative 'misfires' which could profitably have been investigated but which were left to lie.

Turning to psychodynamic elements of the therapy, we hoped to see the use of transference interpretations but found this only in eight sessions; of concern was that the raters thought that evidence of missing transference material was also common in the sessions $(n=13)$.

Variations in skill in handling of different aspects of therapy was associated with the background orientation of the therapist. Therapists from cognitive backgrounds were most active and showed most skill in handling the more cognitive parts of the therapy, such as setting homework, but they tended to miss transference material. A few of the therapists with a cognitive background seemed to keep only to cognitive therapy and avoided psychodynamic and emotional issues.

Therapists from a psychodynamic background tended to make errors in areas with a cognitive 
Table 1. Elements of CAT judged in the audit

1. The type of interpretive interventions made

In CAT there are written agreed foci for therapy. Interventions were categorised as either definite (l.e. explicitly referring to the foci) or vague (l.e. not explicitly linked to the focus of therapy).

2. Use of transterence interpretations

Transference interpretations were defined as any interpretation or description by the therapist of the patient's experience or behaviour which linked aspects of this outside the consulting room (either in the present or the past) with similar situations in the therapeutic situation.

3. Evidence of 'missed transference'

This feature referred to the rater's judgement that the patient was expressing or acting out in the session material which clearly had a transference meaning and this material was not overtly responded to in that session by the therapist.

4. The presence or absence of 'feecback'

This related to the actions of the therapist who has made an intervention which seems to have 'misfired' in some way (e.g. not been understood). Feedback by the therapist was any intervention aimed at checking, acknowledging or righting the 'misfire'.

5. Evidence of 'missed affect'

Missed affect was categorised as the impression on the part of the raters that the therapist was responding in a way which indicated a significant lack of rapport by the therapist with the dominant affect being expressed by the patient.

\section{Background orientation of the therapist}

This referred to the basic initial training or predominant initial theoretical orientation of the therapist before entering the CAT training.

7. Experience of thercaplst

Level of experience as a CAT therapist is marked by promotion to 'supervisor' status. Thus therapists could be categorised in terms of CAT experience as either at supervisor or below supenvisor level.

8. Dificulty of the patient

As part of the ongoing general handling of case allocation in the clinic which the therapists were drawn from patients were routinely judged on the basis of the referral letter to fall into elther a 'standard' or a 'needs experienced therapist (NET)' group.

flavour, such as setting homework, and they tended to be less active overall. Although they did use transference interpretations, a group of them surprisingly tended to miss the predominant emotional tone. Impressionistically, this disappointing result was partly due to a group of rather inexperienced therapists with psychodynamic backgrounds who tended to make transference interpretations when they felt they were in trouble. Consequently they often responded to patient distress at a minor 'miss' in emotional rapport with a transference interpretation which was inaccurate and repeated the 'miss', thus increasing the patient's distress and sometimes starting the first round of a cycle of diminishing rapport.

Intriguingly, the highest rate of transference interpretations is scored by the three therapists who had a purely CAT background. This may result from a combination of the emphasis on activity in the context of a brief focal therapy with an explicit expectation that transference interpretations are valuable and should be used which is also part of CAT therapy.

Overall, therapists demonstrated a variety of therapeutic blunders. These ranged from collusions through failures of empathy to behaviour which seemed on one tape to border on a complete loss of control by one therapist. Where therapists had shown very severe blunders on tape we asked the supervisors for their independent assessment of their trainees' handling of the cases. It was clear that often major blunders were not reported to supervisors who, it seemed, could be unaware of some important aspects of the poor quality of their trainees' performances in these cases. Some supervisors reported doubts based on the performance of trainee therapists in supervision but had not taken further action on the grounds that it was difficult to know what was really going on in therapy.

Finally, it was clear that some patients, especially the 'needs experienced therapist' group. represented a major therapeutic challenge to their therapist. Experienced therapists were often beaten back and trainee therapists could be terrorised or neutralised. Sometimes psychiatric management seemed more appropriate as it was unclear what was being achieved other than completing a 16 session therapy for form's sake.

\section{Conclusions - responses to our audit}

Following our review of these results we felt able to make a number of recommendations for the treatment and training programmes at the clinic. These points were fed back to the treatment team 
both in formal meetings and at frequent informal contacts. Action was taken by the organisers of the service on many of the points raised.

\section{Treatment issues}

Re-evaluation of the benefits and drawbacks of therapeutic heroism. Some cases seemed to be too difficult for treatment in this setting or to have been allocated to a junior trainee.

Action - more emphasis was placed on the need for a specifically psychotherapeutically informed initial assessment (which was not often done) and it was decided that all patients should receive this before being accepted for CAT therapy. Possible increased use of trial of therapy would be attempted, ie. four sessions given first to see how things will go.

\section{Training issues}

(a) CAT requires a range of skills. Therapists coming from different backgrounds require training focused at their weaknesses and may not be learning to strengthen these areas in a generally focused course.

Action-design and introduction of a modular teaching course with increased emphasis on cognitive aspects of the therapy. Students to have a personal tutor to help them identify personal strengths and weaknesses. Evaluation of tapes of therapy made a course requirement.

(b) CAT training needs to recognise that not all therapists initially selected as suitable prove to be so. A clear monitoring and evaluation of therapist quality is essential to reduce the rate of major therapeutic blunders.

Action - introduction of a more formal training course with course criteria and supervisors reports.

\section{Supervision issues}

A major rethink of supervision practice is needed since it is clear that supervisors are not in touch with the strengths and weaknesses of some of the trainees.

Action - widespread recognition of the need for increased supervisor training and of the nature of supervision as a skilled task in its own right. Setting up of the CAT enhancement project. A special group of experienced therapists who will receive more intensive supervision and in which the supervision of tape segments from therapy sessions will be piloted.

\section{General discussion}

It was surprising that an informal and relatively unplanned audit should have had a comparatively large effect on organisational thinking in the department when previous audit had not done so. Indeed, it is argued (Mugford et al, 1991) that an audit like ours - unstructured and conducted by people other than the clinicians themselves - is least likely to be effective in closing the audit cycle.

The use of tapes may have been responsible in part. In our audit they permitted a detailed review of the difficulties encountered by the therapists and allowed delineation of common problems which was appreciated by therapists, often with audible sighs of relief that others were having similar problems.

It is also possible that some of the impact of the audit was due to the use of rated items which were closely related to the real concerns of therapists. While supervision represented the most regular audit of quality in the department, it is possible that our audit had advantages over that process because it was more distant from the ongoing concerns of each therapy and was able to take an overview, allowing teachers, supervisors and therapists to air their concerns about therapy practice in a less personal way.

\section{References}

Feldman, M.M. (1992) Audit in psychotherapy: the concept of Kaizen. Psychiatric Bulletin. 16, 334-335.

FonAGY, P. \& HigGrT, A. (1989) Evaluating the performance of departments of psychotherapy. Psychoanalytic Psychotherapy. 4, 121-153.

MUgFoRd, M., BANFIEld, P. \& O'HANLON, M. (1991) Effects of feedback of information on clinical practice: A review. British Medical Journal, 303, 398-402.

PARRY, G. (1992) Improving psychotherapy services: applications of research, audit and evaluation. British Journal of Clinical Psychology, 31, 3-19.

RYLE, A. (1990) Cognitive-Analytic Therapy: active partictpation in change. Chichester: Wiley.

WATSON, J.P. \& RYLE, A. (1992) Providing a psychotherapy service to an inner London catchment area. Journal of Mental Health, 1, 169-176.

F. Denman, Senior Registrar in Psychotherapy, The Cassel Hospital, 1 Ham Common TW10 7JF 